Originalien

Z Gerontol Geriat 2022 · 55:223-230 https://doi.org/10.1007/s00391-021-01841-7 Eingegangen: 13. März 2020

Angenommen: 30. Dezember 2020

Online publiziert: 26. Januar 2021

(c) Der/die Autor(en) 2021

Fabian Moser ${ }^{1,2} \cdot$ Leonhard H. Schütz ${ }^{2} \cdot$ Christian Teubner $^{1} \cdot$ Nils Lahmann $^{3}$. Adelheid Kuhlmey ${ }^{2} \cdot$ Ralf Suhr $^{1}$

'Stiftung Zentrum für Qualität in der Pflege, Berlin, Deutschland

${ }^{2}$ Institut für Medizinische Soziologie und Rehabilitationswissenschaft, Charité Universitätsmedizin Berlin, Berlin, Deutschland

${ }^{3}$ Klinik für Geriatrie und Altersmedizin, Charité Universitätsmedizin Berlin, Berlin, Deutschland

\title{
Sexueller Missbrauch Pflegebedürftiger
}

\section{Ergebnisse einer bundesweiten Querschnittsstudie bei Hausärzten/ -ärztinnen zu Verantwortung und subjektiven Sicherheit im Verdachtsfall}

häufigste Gewaltform. Finanzielle Ausbeutung stellt mit 6,8 \% die zweithäufigste Form dar, gefolgt von Vernachlässigung $(4,2 \%)$ und körperlicher Gewalt $(2,6 \%)$. Sexueller Missbrauch älterer Menschen ist mit $0,9 \%$ seltener.

Die Folgen von Gewalt gegen ältere Menschen sind vielfältig und für Betroffene sowie das Gesundheitssystem bedeutsam. So steigt bei Menschen, die Gewalt gegen sich erfahren haben, beispielsweise das Sterberisiko, sie weisen eine höhere Rate an Depressionen auf, und es kommt vermehrt zu Hospitalisierungen $[2,19,20]$.

Pflegebedürftigkeit bzw. physische oder kognitive Einschränkungen sind Risikofaktoren für Gewalt im Alter [25]. Repräsentative Daten zur Prävalenz von Gewalt gegen Pflegebedürftige in Deutschland liegen nicht vor. Verschiedene Studien zeigen jedoch, dass Gewalt gegen Pflegebedürftige in der häuslichen, ambulanten und stationären Langzeitpflege in Deutschland als relevantes Problem wahrgenommen wird und häufig auftritt [7]. Viele pflegewissenschaftliche Untersuchungen befassen sich daher auch mit Ursachen, Auslösern und Möglichkeiten zur Prävention von Gewalt im Kontext Pflege [4]. In bestimmten Fällen, wie bei den freiheitsentziehenden Maßnahmen (FEM), führten diese Bestrebungen zu konkreten pflegerischen Leitlinien und Interventionen zur Gewaltprävention. Sexuelle Gewalt gegen Pflegebedürftige - also die erzwungene Teilnahme einer älteren, pflegebedürftigen Person an einer sexuellen Handlung ohne deren Einwilligung [32] - ist bisher kaum untersucht [3].

Im Zusammenhang mit dem Erkennen und der Prävention von Gewalt gegen Pflegebedürftige sprechen die Breite, Frequenz und Qualität des Arzt-PatientKontakts auch für eine mögliche Rolle von Hausärzten/-ärztinnen. So geben über drei Viertel der 60- bis 79-jährigen an, innerhalb der letzten 12 Monate mindestens einmal Kontakt zu ihren Hausärzten/-ärztinnen gehabt zu haben [15]. Die Arzt-Patient-Beziehung ist zudem durch ein Vertrauensverhältnis gekennzeichnet, welches es erlaubt, auch sensible und intime Fragen zu besprechen [8]. Das Potenzial von Hausärzten/-ärztinnen beim Erkennen und bei der Prävention von Gewalt gegen ältere Menschen wird hierbei durch die internationale Forschung gestützt [24]. In einer Studie in Irland wurde gezeigt, dass die meisten Fälle von Gewalt gegen Ältere durch Hausärzte/-ärztinnen in der Häuslichkeit aufgedeckt werden [24]. In einer Befragung von Hausärzten/-ärztinnen in Ohio, USA, gab der Großteil der Befragten an, Hausärzte/-ärztinnen seien unterscheiden sich deutlich [31]. Psychische Misshandlung ist mit 11,6\% die 
besser in der Lage, Gewalt zu erkennen, als andere Gesundheitsprofessionen [16].

Das Thema findet anamnestisch in der Hausarztpraxis jedoch keine systematische Berücksichtigung; eine routinemäßige Befragung älterer Patienten/ Patientinnen zu erfahrener Gewalt führen nur jeder/jede siebte Arzt/Ärztin durch [5]; über drei Fünftel befragen diese nie oder fast nie dazu [16]. Auch wird nur ein geringer Anteil der Verdachtsfälle gemeldet [5, 24]. Als ein Grund wird angeführt, dass Ärzte/ Ärztinnen sich erst vollständig sicher sein wollten, bevor sie einen Verdacht anzeigten [16]. Knappe zeitliche Ressourcen dürften ebenfalls eine Rolle spielen: Rund zwei Drittel (62\%) der deutschen Hausärzte/-ärztinnen geben an, nicht ausreichend Zeit für die Behandlung ihrer Patienten/Patientinnen zu haben [13]. Zudem schätzen internationale Studien das ärztliche Wissen zu Gewalt gegen Ältere als gering ein, und es wird auf Fortbildungsbedarf hingewiesen [5, 16].

Für Deutschland liegen keine wissenschaftlichen Befunde zum Wissen von Hausärzten/-ärztinnen oder zu ihrer Rolle beim Erkennen und der Prävention von Gewalt gegen Pflegebedürftige vor. Vor allem gibt es - auch international - keine Untersuchung, die explizit die Frage der von Hausärzten/-ärztinnen wahrgenommenen Verantwortung bei sexuellem Missbrauch pflegebedürftiger Patienten/Patientinnen beleuchtet.

Forschungsfragen dieser Arbeit sind:

1. Wo sehen deutsche Hausärzte/-ärz-

tinnen ihren Verantwortungsbereich beim Erkennen und der Vorbeugung von sexuellem Missbrauch Pflegebedürftiger?

2. Wie sicher sind sich Hausärzte/-ärztinnen im weiteren Vorgehen bei einem vermuteten Fall von sexuellem Missbrauch Pflegebedürftiger?

\section{Methodik}

\section{Studiendesign und Vorgehen}

In einer bundesweiten Querschnittserhebung wurden Hausärzte/-ärztinnen in Deutschland zwischen September und
November 2016 schriftlich befragt ${ }^{1}$. Um eine angemessene Präzision der Konfidenzintervalle zu gewährleisten, wurde eine Teilnehmerzahl von mindestens 250 angestrebt. Ausgehend von einer angenommenen Rücklaufquote von 15-20\%, basierend auf deutschen Erhebungen bei einem vergleichbaren Befragtenkollektiv [18, 22], wurden Fragebogen an 1700 Teilnehmer*innen gesendet. Die Auswahl der Ärzte/Ärztinnen erfolgte per Zufallsstichprobe aus einer kommerziellen Datenbank [1], die nach eigenen Angaben die Daten nahezu aller (99\%) niedergelassenen Arztpraxen in Deutschland beinhaltet. Zur Erhöhung des Rücklaufs wurde 4 Wochen nach Erstversand ein Erinnerungsschreiben, inklusive Fragebogen, an diejenigen geschickt, die bisher nicht teilgenommen hatten. Zur Identifikation des Rücklaufs waren die Fragebogen mit einem adressatenspezifischen Code versehen. Die Studie wurde von der Kassenärztlichen Bundesvereinigung durch einen Artikel im Newsletter PraxisNachrichten unterstützt, in welchem die besondere Relevanz hervorgehoben wurde. Ein positives Ethikvotum liegt vor (Eth21/16).

\section{Variablen}

Erfasst wurden Alter, Geschlecht und Berufserfahrung sowie der Anteil pflegebedürftiger Patienten/Patientinnen. Als „pflegebedürftig“ wurden erwachsene Personen definiert, die aufgrund von körperlichen, kognitiven oder psychischen Beeinträchtigungen dauerhaft Hilfe durch andere benötigen. Die Datenerhebung $\mathrm{zu}$ Fragen des sexuellen Missbrauchs wurde im Rahmen einer größeren trinationalen Studie parallel in Deutschland, Österreich und der Schweiz mit dem gleichen Fragebogen durchgeführt; daher wurden Verweise auf nationale sozialrechtliche Regelungen vermieden, und ein expliziter Bezug auf die zum Zeitpunkt der Studiendurch-

\footnotetext{
1 Schnapp P (2018) Bericht zur ZQP-Studie Gewalt gegen pflegebedürftige Menschen: Prävention durch Hausärztinnen und Hausärzte. Zentrum für Qualität in der Pflege, Berlin, unveröffentlicht
}

führung gültige deutsche Legaldefinition des SGB XI $\$ 14$ erfolgte nicht.

Daneben wurde erhoben, ob dem/der Hausarzt/-ärztin bei aktuell versorgten pflegebedürftigen Patienten/Patientinnen bekannt sei, dass diese schon einmal Opfer von sexuellem Missbrauch durch pflegende Personen geworden seien.

Sechs Fragen erfassten die Einstellung zur ärztlichen Verantwortung beim Erkennen und Vorgehen gegen sexuellen Missbrauch Pflegebedürftiger. Sexueller Missbrauch wurde hierbei als „sexuelle Handlungen, die an Menschen ohne deren Einverständnis vorgenommen werden“, definiert.

Zur Messung der Sicherheit hinsichtlich des weiteren Vorgehens im Verdachtsfall wurde die Zustimmung zur Aussage gemessen, dass der/die Befragte „unsicher [wäre], wie [er/sie] weiter vorgehen sollte“, wenn er/sie „den Verdacht hätte, dass ein Patient, der pflegebedürftig ist" Opfer von sexuellem Missbrauch geworden wäre.

Zum Fortbildungsinteresse wurden zwei Fragen gestellt, die jeweils bejaht bzw. verneint werden konnten. $\mathrm{Zu}$ nächst wurde das allgemeine Interesse der Teilnehmer*innen an Fortbildung zum Thema des sexuellen Missbrauchs Pflegebedürftiger erfragt. Für Interessierte wurde erhoben, zu welchen einzelnen Themen hierbei Fortbildungsbedarf bestünde.

\section{Bias}

Die Items wurden vor der eigentlichen Studie mithilfe eines mehrstufigen Pretests umfangreich geprüft. In einem ersten Schritt wurde der Fragebogen von $\mathrm{Ge}$ waltexperten hinsichtlich Verständlichkeit der Formulierungen und inhaltlicher Vollständigkeit geprüft und angepasst, dann erfolgte durch weitere Experten eine Begutachtung der Validität der gewählten Items. In einer zweiten Phase wurde zur Bestimmung der Retest-Reliabilität und zur Messung sozial erwünschten Antwortverhaltens der vorläufige Fragebogen an eine Zufallsstichprobe von Hausärzten/-ärztinnen versandt. Wurde der ausgefüllte Fragebogen zurückgesandt, erhielt der/die Befragte einen gleichen zweiten Fragebo- 
gen. Wurde auch dieser ausgefüllt zurückgeschickt, erhielt er/sie einen Gutschein im Wert von $€ 50$, und die RetestReliabilitäten der Items dieser abhängigen Datensätze wurden berechnet. Die Retest-Reliabilitäten waren hoch bis sehr hoch. Die Korrelationen mit Maßen sozial erwünschten Antwortverhaltens wiederum waren insgesamt gering. Befragte, welche an den Pretests teilgenommen haben, wurden nicht mehr in der Hauptstudie befragt. Die Vorstudien und ihre Ergebnisse werden an anderer Stelle im Detail beschrieben ${ }^{2}[26,27]$.

\section{Datenauswertung und statistische Analyse}

Um Adressaten/Adressatinnen, die keine Hausärzte/-ärztinnen sind, von den Analysen ausschließen zu können, wurde erfragt, ob der/die Teilnehmer*in tatsächlich hausärztlich tätig sei. Daneben wurden Fragebogen von der weiteren Auswertung ausgeschlossen, wenn weniger als $80 \%$ der Items beantwortet wurden. Vor der Berechnung deskriptiver Statistiken wurde eine Anpassungsgewichtung auf Grundlage der Informationen des Bundesarztregisters für die Arztgruppen „Allgemeinarzt/-ärztin, praktische Ärztinnen und Ärzte“ sowie „hausärztlich tätige Internisten“ [14] nach Geschlecht und Nielsengebiet [12] vorgenommen. Nielsengebiete resultieren aus einer $\mathrm{Zu}$ sammenfassung einzelner Bundesländer in 7 in ihrem Konsummuster vergleichbare Gebiete und wurden aufgrund der kleinen Stichprobengröße, statt der einzelnen Bundesländer, für die Gewichtung herangezogen.

Zur Auswertung der gewichteten Daten wurden Mittelwerte und 95\%Clopper-Pearson-Konfidenzintervalle bestimmt. Zur statistischen Auswertung wurde SPSS ${ }^{\circledR}$ genutzt.

\footnotetext{
2 Schnapp P (2018) Bericht zur ZQP-Studie Gewalt gegen pflegebedürftige Menschen: Prävention durch Hausärztinnen und Hausärzte. Zentrum für Qualität in der Pflege, Berlin, unveröffentlicht
}

Z Gerontol Geriat 2022 ·55:223-230 https://doi.org/10.1007/s00391-021-01841-7

(C) Der/die Autor(en) 2021

F. Moser · L. Schütz · C. Teubner · N. Lahmann · A. Kuhlmey · R. Suhr

\section{Sexueller Missbrauch Pflegebedürftiger. Ergebnisse einer bundesweiten Querschnittsstudie bei Hausärzten/-ärztinnen zu Verantwortung und subjektiven Sicherheit im Verdachtsfall}

\section{Zusammenfassung}

Hintergrund. Gewalt gegen pflegebedürftige ältere Menschen ist häufig. Hausärzte/ärztinnen können bei der Prävention von Gewalt eine Rolle spielen. Insbesondere sexualisierte Gewalt ist stark tabuisiert und wenig untersucht.

Ziel der Arbeit. Ziel dieser Arbeit ist es, die Einstellung von Hausärzten/-ärztinnen zu ihrer Verantwortung bei sexuellem Missbrauch pflegebedürftiger Patienten/Patientinnen zu untersuchen. Zugleich sollen die subjektive Sicherheit hinsichtlich des Vorgehens bei einem Missbrauchsverdacht sowie die Fortbildungsinteressen von Hausärzten/ärztinnen zum Thema erhoben werden. Material und Methoden. In einer Querschnittsstudie wurden 1700 Hausärzte/ärztinnen in Deutschland zwischen September und November 2016 schriftlich befragt. Fragebogen von 302 Ärzten/Ärztinnen konnten ausgewertet werden.
Ergebnisse. Die Unsicherheit hinsichtlich des weiteren Vorgehens bei Verdacht auf sexuellen Missbrauch von pflegebedürftigen Patienten/Patientinnen ist groß. Nahezu alle Befragten sehen es als Teil der ärztlichen Verantwortung, bei sexuellem Missbrauch pflegebedürftiger Patienten/Patientinnen zu intervenieren. Hauptsächliches Fortbildungsinteresse besteht zur Differenzialdiagnose des sexuellen Missbrauchs sowie zum richtigen Vorgehen im Verdachtsfall.

Schlussfolgerung. Fortbildungen, insbesondere zu den Anzeichen sexueller Gewalt gegen Pflegebedürftige, können einen Beitrag leisten, die Handlungssicherheit von Hausärzten/-ärztinnen zu stärken und ihre Bereitschaft zur Prävention zu erhöhen.

Schlüsselwörter

Sexuelle Gewalt · Pflegebedürftigkeit . Hausarzt $\cdot$ Verantwortung $\cdot$ Missbrauch

\section{Sexual abuse of care-dependent patients. Results of a nationwide cross-sectional study among general practitioners on responsibility and subjective confidence in dealing with suspected abuse}

Abstract

Background. Violence against people in need of care is a challenge for long-term care situations. Sexual violence in particular, is subject to strong taboos and has so far been little researched. Family physicians can play a role in preventing violence against people in need of care.

Objective. The objective of this study was to examine family physicians' attitudes to their responsibilities in cases of sexual abuse of patients in need of care. At the same time, we also examined subjective confidence in relation to the procedure in cases of suspected abuse and family physicians' interests in further training on this topic. Material and methods. A cross-sectional study addressed to 1700 family physicians in Germany with a written survey between September and November 2016. Questionnaires from 302 physicians could be evaluated for the study.
Findings. Almost all respondents saw it as part of their responsibility as doctors to intervene in cases of sexual abuse of patients in need of care. There is great uncertainty about how to proceed in cases of suspected sexual abuse of patients in need of care. Respondents' main interest in further training related to the differential diagnosis of sexual abuse and the correct procedure in cases where abuse is suspected.

Conclusion. Further training provision, particularly on the signs of sexual abuse of people in need of care, could contribute to increasing family physicians' confidence to act.

Keywords

Sexual violence $\cdot$ Need of care $\cdot$ Family physician · Responsibility · Abuse 
Wenn ein HA erfährt, dass ein pflegebed. Pat. Opfer sex. Missbrauchs geworden ist, muss er alles dafür tun, dass sich das nicht wiederholt.

Ein HA muss versuchen, weiteren sex. Missbrauch gegen einen pflegebed. Pat. zu verhindern, auch wenn hierdurch Konflikte zwischen dem Arzt und den pflegenden Personen entstehen.

Wenn ein HA erfährt, dass eine pflegende Person eine pflegebed. Person sex. missbraucht, muss der HA dagegen vorgehen.

Es gehört zu den Pflichten eines HA, gegen sex. Missbrauch in Pflegebeziehungen seiner Pat. vorzugehen.

Ein $\mathrm{HA}$ ist verantwortlich dafür, gegen die Ursachen sex. Missbrauchs pflegebed. Pat. vorzugehen.

Ein HA sollte pflegebed. Personen grundsätzl. daraufhin untersuchen, ob sie zum Opfer sex. Missbrauchs geworden sind, auch wenn es hierfür keine Anzeichen gibt.

0 [stimme überhaupt nicht zu]

1

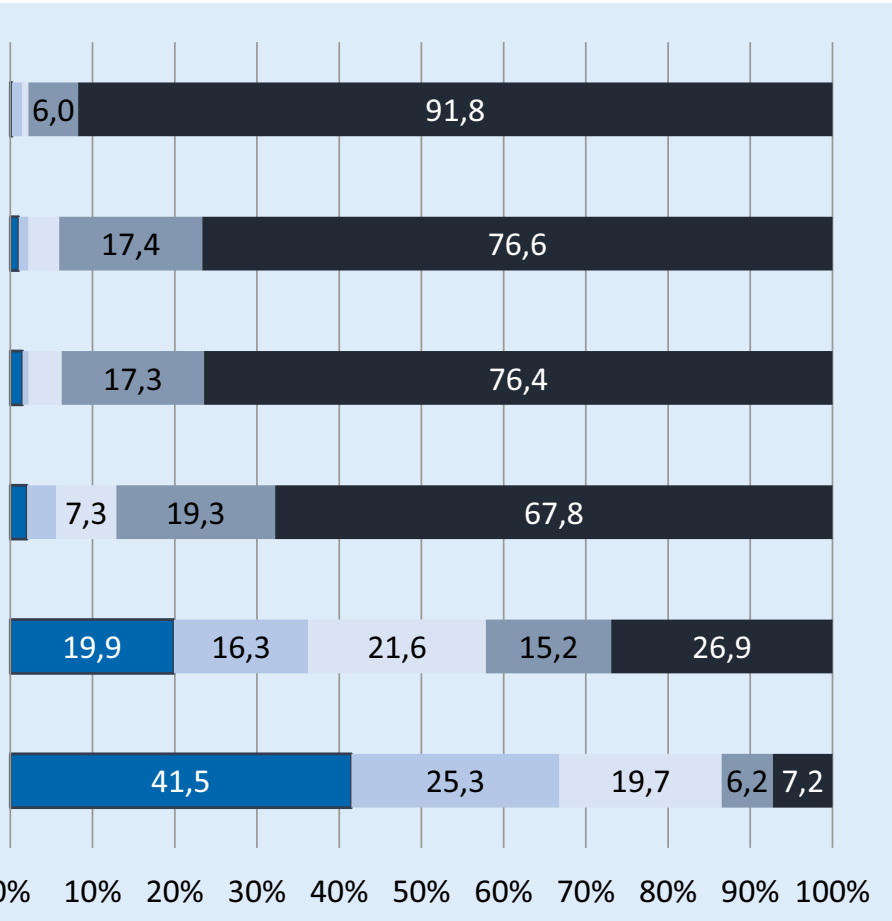

$2 \quad 3$

4 [stimme voll und ganz zu]

Abb. 1 ॥ Einstellungen der Hausärzte/-ärztinnen (HA) zum Umgang mit sexuellem Missbrauch pflegebedürftiger Patienten/ Patientinnen $(n=302)$. Dargestellt ist der originale Wortlaut des Fragebogens

\section{Ergebnisse}

\section{Rücklauf und Soziodemografie}

Die Rücklaufquote beträgt 18,1\%: 302 Fragebogen konnten ausgewertet werden; in 4 Fällen wurden von dem/der teilnehmenden Hausarzt/-ärztin weniger als $80 \%$ der Items ausgefüllt, in weiteren 2 Fällen wurde eine Teilnahme verweigert bzw. abgebrochen. Aus dem Gesamtrücklauf (inkl. der Fälle, die nachweislich nicht oder nicht mehr hausärztlich tätig waren) wurde ein Anteil von $89 \%$ unter den Nonrespondern geschätzt, welcher das Teilnahmekriterium erfüllt. Eine weitergehende Nonresponderanalyse wurde nicht durchgeführt. Der erhobene Rohdatensatz weist bereits ungewichtet eine sehr große Übereinstimmung mit der Grundgesamtheit der deutschen hausärztlich tätigen Ärzte/ Ärztinnen auf. Es geben 43,1\% der Befragten an weiblichen Geschlechts $\mathrm{zu}$ sein, 56,9\% sind Männer. Das Durchschnittsalter beträgt 55,7 Jahre [54,7; 56,6]. Die Studienteilnehmer ${ }^{\star}$ innen ge- ben an, im Durchschnitt 18,6 Jahre [17,5; 19,7] als Hausarzt/-ärztin niedergelassen $\mathrm{zu}$ sein. Durchschnittlich 10,9\% $[9,8 ; 12,0]$ der Patienten/Patientinnen der befragten Hausärzte/-ärztinnen sind pflegebedürftig. Acht Hausärzte/-ärztinnen geben an, von sexuellem Missbrauch bei mindestens einem ihrer pflegebedürftigen Patienten/Patientinnen zu wissen.

\section{Einstellung der Hausärzte/- ärztinnen zur Verantwortung bei sexuellem Missbrauch}

Nahezu alle Befragten (91,8\%) stimmen „voll und ganz zu“, dass Hausärzte/-ärztinnen alles dafür tun müssen, um zu verhindern, dass sich ein sexueller Missbrauch wiederholt (• Abb. 1). Dem stimmen noch $76,6 \% \mathrm{zu}$, wenn durch die Intervention Konflikte zwischen Hausärzten/-ärztinnen und den pflegenden Personen hervorgerufen würden. Rund zwei Drittel der Befragten sehen es „voll und ganz“ als hausärztliche Pflicht, gegen sexuellen Missbrauch pflegebedürftiger Patienten vorzugehen; deutlich weni- ger $(26,9 \%)$ sehen sich uneingeschränkt in Verantwortung, gegen dessen Ursachen vorzugehen. Am geringsten fällt die Zustimmung zur grundsätzlichen Untersuchung pflegebedürftiger Patienten/ Patientinnen auf sexuellen Missbrauch aus (7,2\%). Es bestehen keine statistisch signifikanten Zusammenhänge zu Strukturvariablen wie Alter, Geschlecht oder Niederlassungsdauer.

\section{Unsicherheit bezüglich des weiteren Vorgehens}

Von den befragten Hausärten/-ärztinnen äußern 26,5\% keine Unsicherheit hinsichtlich des weiteren Vorgehens im Verdachtsfall, weitere $22,7 \%$ äußern geringe Unsicherheit. Die restlichen Befragten stimmen der Aussage in unterschiedlichem Maße zu, bei Verdacht auf sexuellen Missbrauch unsicher bezüglich des weiteren Vorgehens zu sein. Hier zeigt sich ein statistisch signifikanter, jedoch sehr schwach positiver Zusammenhang zwischen der geäußerten subjektiven Sicherheit und dem Alter $(r=0,21, p<0,01)$, der 


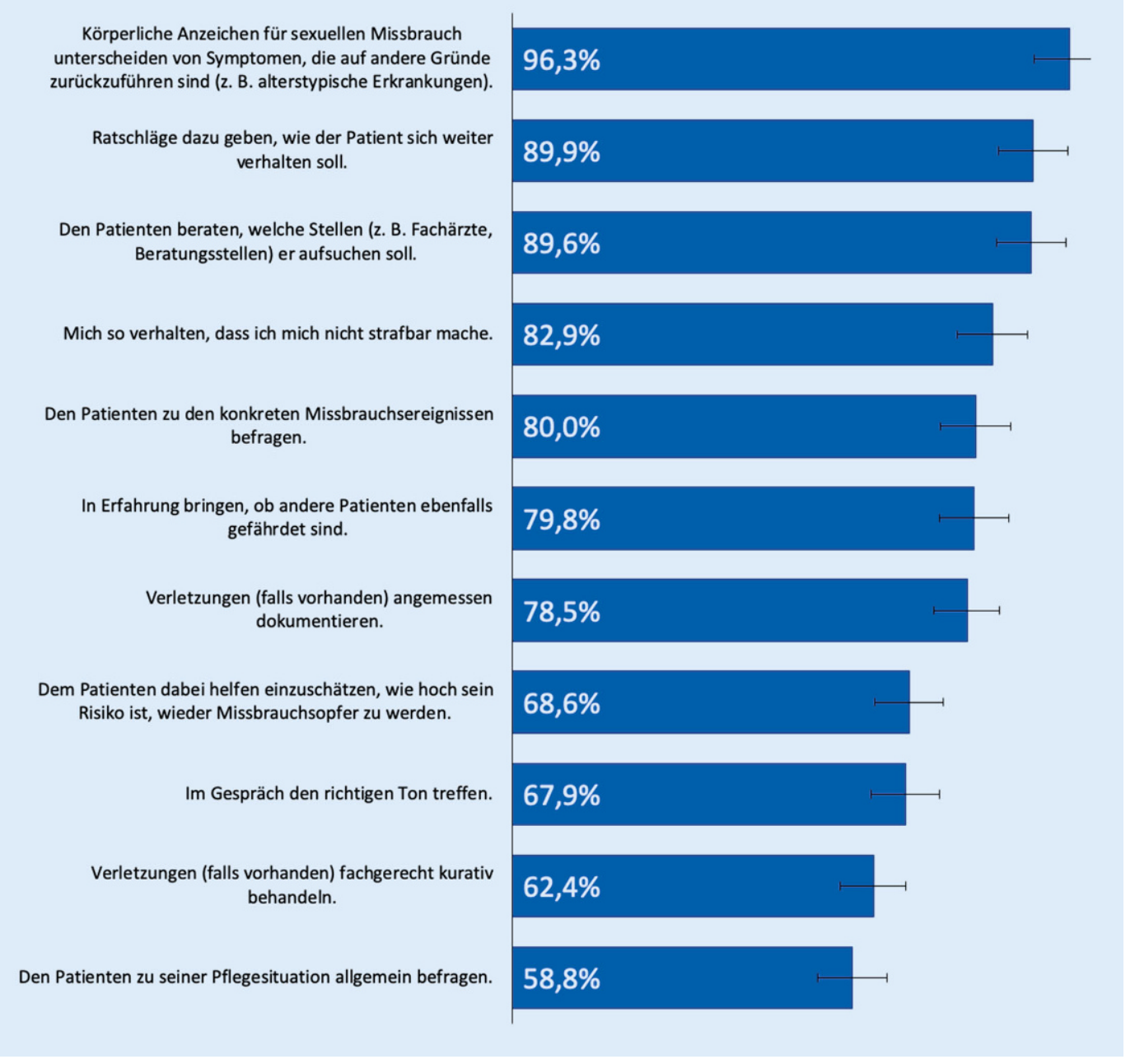

Abb. $2 \triangleleft$ Interesse für ein bestimmtes Fortbildungsthema bei Hausärzten/-ärztinnen, die Fortbildungsinteresse haben (Anteile und $95 \%$-Konfidenzintervall). Dargestellt ist der originale Wortlaut des Fragebogens
Niederlassungsdauer $(\mathrm{r}=0,19, p<0,01)$ und dem Geschlecht $(\mathrm{r}=0,11, p<0,05)$ der Befragten.

\section{Interesse an Fortbildungen zum Thema sexueller Missbrauch}

Knapp die Hälfte der Studienteilnehmer*innen $(48,2 \%)$ gibt ein Interesse an Fortbildungen zum Thema „sexueller Missbrauch Pflegebedürftiger“ an. Diese Gruppe wurde genauer dazu befragt, welche Fortbildungsinhalte sie hierbei präferiert (• Abb. 2).

Fast alle Fortbildungsinteressierten $(96,3 \%[91,6 ; 98,7] ; n=139)$ geben an, sich zum Erkennen von Symptomen des sexuellen Missbrauchs weiterbilden $\mathrm{zu}$ wollen. Neun von 10 Befragten (89,9\% $[83,6 ; 94,5] ; n=134)$ zeigen Interesse an Fortbildungen zu Ratschlägen, wie der Patient/die Patientin sich weiter verhalten soll bzw. zur Beratung, welche Stellen er/sie aufsuchen soll $(89,6 \%$ [83,2; 94,2], $n=137)$. Geringer ausgeprägt ist das Interesse an Fortbildungen zur allgemeinen Befragung von Patienten/Patientinnen $\mathrm{zu}$ deren Pflegesituation (58,8\% [50,0; 67,1], $n=135)$.

\section{Diskussion}

In vorliegender Arbeit wurde erhoben, in welchem Maß Hausärzte/-ärztinnen es als ihre Verantwortung ansehen, gegen sexuellen Missbrauch pflegebedürftiger Patienten/Patientinnen vorzugehen. $\mathrm{Zu}$ gleich wurden die subjektive Sicherheit zum Vorgehen im Verdachtsfall sowie das Weiterbildungsinteresse untersucht.

Nahezu alle Befragten sehen es als Teil der ärztlichen Verantwortung, bei Bekanntwerden des sexuellen Missbrauchs zu intervenieren - selbst auf die Gefahr hin, dass hierdurch Konflikte beispielweise mit den Pflegepersonen entstehen wür- den. Gegen Ursachen vorzugehen und eine damit stärker präventiv ausgerichtete hausärztliche Verantwortung werden von den in dieser Studie Befragten mehrheitlich verneint.

Sicherlich könnten in dieser Hinsicht auch weitere Gruppen eine wichtige Rolle spielen, wie beispielsweise die Pflegeberatung oder ein Pflegedienst. Durch ihren häufigen regelmäßigen Kontakt zu pflegebedürftigen Personen und dadurch, dass sie in deren körperliche Pflege einbezogen sind, können sehr frühzeitig Anzeichen von Gewalt erkannt werden. Als mögliche Einschränkungen sind hier jedoch zu nennen, dass über zwei Drittel der in der Häuslichkeit lebenden Pflegebedürftigen in Deutschland ausschließlich von pflegenden Angehörigen versorgt werden [28]. Sie haben somit keinen regelmäßigen Kontakt zu professionell Pflegenden. Zugleich kann 
mitunter von professionell Pflegenden selbst Gewalt ausgeübt werden [4].

Die wenigen vorliegenden internationalen Untersuchungen unterstreichen - allgemein im Kontext der Intervention bei Gewalt gegen Ältere - eine wichtige hausärztliche Rolle [24] und zeigen das hausärztliche Verantwortungsbewusstsein [16] auf. Für eine Verantwortung von Hausärzten/-ärztinnen bei der Intervention gegen sexuellen Missbrauch pflegebedürftiger Patienten/Patientinnen spricht auch die Dichte ihres Patientenkontakts. So sind hausärztlich tätige Allgemeinmediziner ${ }^{*}$ innen die im Erwachsenenalter bei pflegebedürftigen Patienten/Patientinnen am häufigsten aufgesuchten Fachärzte/-ärztinnen [17]. Die meisten Patienten/Patientinnen geben zudem an, ein besonderes Vertrauensverhältnis zu ihren Hausärzten/ärztinnen zu haben [6], wodurch auch besonders sensible und intime Themen besprochen werden können. Gerade angesichts des starken Tabus des sexuellen Missbrauchs [3] könnte dieses besondere Vertrauensverhältnis in der HausarztPatient-Beziehung die Offenlegung von Missbrauchsereignissen fördern.

Gründe für die mehrheitliche Verneinung einer grundsätzlichen Untersuchung Pflegebedürftiger auf Anzeichen sexuellen Missbrauchs wurden in vorliegender Befragung nicht erfasst. Die Ablehnung könnte in den begrenzten zeitlichen Ressourcen [11, 13] von Hausärzten/-ärztinnen begründet liegen. Hinzu kommt, dass es sich um ein stark tabuisiertes Thema handelt. Auch der Großteil der von Kennedy [16] befragten Hausärzte/-ärztinnen erhebt nie oder fast nie systematisch die von Patienten/ Patientinnen erfahrene Gewalt. Weitere Vergleichsstudien zur Einordnung der Ergebnisse liegen aktuell nicht vor.

Die Vorbehalte gegenüber einer Routineuntersuchung könnten darüber hinaus durch Unsicherheit und Wissensdefizite $\mathrm{zu}$ den notwendigen weiteren Schritten bedingt sein. Internationale Studien $[16,21]$ weisen auf Wissenslücken und Fortbildungsbedarf hin. So äußerten beispielsweise in der Studie von McCreadie [23] rund $70 \%$ der befragten Hausärzte/-ärztinnen Fortbildungsbedarf. Für Deutschland gibt es bisher weder Erkenntnisse zum Wissen von Hausärzten/-ärztinnen zu Gewalt gegenüber Pflegebedürftigen im Allgemeinen noch $\mathrm{zu}$ Fragen des sexuellen Missbrauchs im Speziellen.

Die Ergebnisse der vorliegenden Untersuchung machen Wissenslücken plausibel. So äußert lediglich ein gutes Viertel der Befragten, hinsichtlich des weiteren Vorgehens bei Verdacht auf sexuellen Missbrauch sicher zu sein. Zugleich gibt knapp die Hälfte der Befragten allgemeines Interesse an Fortbildungen zum Thema an. Von nahezu allen wird Fortbildungsbedarf zu den körperlichen Anzeichen sexuellen Missbrauchs und deren Abgrenzung zu Symptomen anderer Ursache genannt. Zugleich zeigt sich die Unsicherheit auch darin, dass nahezu alle an Fortbildung Interessierten angeben, sich zu Fragen des richtigen Verhaltens bei festgestelltem Missbrauch und zu relevanten Beratungsstellen fortbilden $\mathrm{zu}$ wollen.

Tilden et al. [29] konnten zeigen, dass durch Wissensvermittlung und gezieltes Training von Ärzten/Ärztinnen zum Vorgehen bei Gewalt gegen ältere Menschen deren Handlungskompetenz erhöht wurde. In dieser Hinsicht könnten gezielte Fortbildungen einen Beitrag leisten, die bestehende Unsicherheit auch bei deutschen Hausärzten/-ärztinnen zu reduzieren. Vorliegende Arbeit gibt Hinweise auf hausärztlichen Fortbildungsbedarf. Sie hat darin praktischen Nutzen für die Entwicklung von Fortbildungsangeboten, die den inhaltlichen Präferenzen der potenziellen Nutzer*innen entsprechen. Die Entwicklung von Fortbildungsinterventionen und die Prüfung ihrer Wirksamkeit zur Steigerung von Wissen und Handlungskompetenz von Hausärzten/-ärztinnen bedürfen weiterer Forschung.

\section{Limitationen}

Die in der vorliegenden Arbeit erfassten Einstellungen können ein guter Prädiktor für tatsächliches Verhalten sein [9]. Die erhobenen subjektiven Einschätzungen lassen jedoch nur eingeschränkt Aussagen zur tatsächlichen Handlungskompetenz sowie zu vorhandenen objektiven Wissenslücken von
Hausärzten/-ärztinnen zu. Trotz umfassender Prüfung und Auswahl der eingesetzten Items [26] könnte sozial erwünschtes Antwortverhalten dazu geführt haben, dass die eigene Sicherheit hinsichtlich des Vorgehens durch die teilnehmenden Ärzte/Ärztinnen tendenziell eher überschätzt wurde. Zudem kann nicht ausgeschlossen werden, dass eher Hausärzte/-ärztinnen mit Interesse am oder Berührung mit dem Thema teilgenommen haben. Hierdurch würden das tatsächliche Wissen von Hausärzten/ärztinnen in Deutschland und deren Fortbildungsinteresse zum Thema überschätzt. Auch eine fehlende Aktualität der Datensätze der ArztData AG könnte zu einem Selektionsbias geführt haben; dieser erscheint jedoch angesichts des geringen Rücklaufs an unzustellbaren Fragebogen eher unwahrscheinlich. Die hohe Übereinstimmung der Daten der Stichprobe mit der Grundgesamtheit kann als Hinweis auf deren externe Validität gewertet werden, auch wenn abschließend kein absolutes $\mathrm{Maß}$ an Repräsentativität genannt werden kann. Aufgrund des Fehlens von Vergleichsdaten aus anderen Studien müssen die Ergebnisse vorliegender Querschnittsstudie durch zukünftige Erhebungen bestätigt und fortgeführt werden.

\section{Fazit}

Die Befragungsergebnisse geben Hinweise auf Unsicherheit und Fortbildungsbedarf von Hausärzten/-ärztinnen zum Thema des sexuellen Missbrauchs Pflegebedürftiger. Aufgrund der besonderen Vertrauensstellung und des häufigen Kontakts zu Pflegebedürftigen stellt sich die hausärztliche Schulung zum Thema als sinnvoll dar. Insbesondere sollten bei Fortbildungsmaßnahmen die Differenzialdiagnose des sexuellen Missbrauchs Pflegebedürftiger sowie das Vorgehen im Verdachtsfall Berücksichtigung finden.

\section{Fünf Kernaussagen}

1. Hausärzte/-ärztinnen sehen es als ihre Pflicht, bei vermutetem sexuellem Missbrauch eines Pflegebedürftigen $\mathrm{zu}$ intervenieren.

2. Eine Routineuntersuchung Pflegebedürftiger auf Anzeichen se- 
xuellen Missbrauchs wird von Hausärzten/-ärztinnen abgelehnt.

3. Ein Großteil der befragten Hausärzte/-ärztinnen äußert Unsicherheit hinsichtlich des Vorgehens bei Verdacht auf sexuellen Missbrauch Pflegebedürftiger.

4. Die Hälfte der befragten Hausärzte/ -ärztinnen wünscht sich Fortbildungen zum Thema des sexuellen Missbrauchs Pflegebedürftiger.

5. Besonderer Fortbildungsbedarf besteht hinsichtlich der Differenzialdiagnose des sexuellen Missbrauchs und der Abgrenzung von Missbrauchsanzeichen zu alterstypischen Veränderungen.

\section{Korrespondenzadresse}

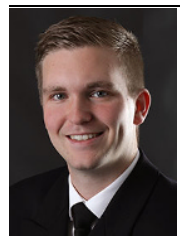

Fabian Moser

Stiftung Zentrum für Qualität in der Pflege

Reinhardtstr. 45, 10117 Berlin, Deutschland

Fabian-a.Moser@charite.de

Danksagung. Die Autoren danken Frau Prof. A. Berzlanovich für deren Beratung bei der Studienkonzeption, Herrn Dr. P. Schnapp für seine Arbeit bei der Entwicklung des Erhebungsinstruments und der Durchführung der Befragung sowie Frau B. Schaefer für das Lektorat des englischsprachigen Abstracts.

Funding. Open Access funding enabled and organized by Projekt DEAL.

\section{Einhaltung ethischer Richtlinien}

Interessenkonflikt. F. Moser, L. Schütz, C. Teubner, N. Lahmann, A. Kuhlmey und R. Suhr geben an, dass kein Interessenkonflikt besteht. R. Suhr ist Vorstandsvorsitzender des ZQP, einer gemeinnützigen Stiftung bürgerlichen Rechts, die 2009 durch den Verband der Privaten Krankenversicherung e. V. gestiftet wurde.

Ein positives Ethikvotum der Ärztekammer Berlin liegt vor. Es wurden keine Versuche an Menschen oder Tieren durchgeführt.

Open Access. Dieser Artikel wird unter der Creative Commons Namensnennung 4.0 International Lizenz veröffentlicht, welche die Nutzung, Vervielfältigung, Bearbeitung, Verbreitung und Wiedergabe in jeglichem Medium und Format erlaubt, sofern Sie den/die ursprünglichen Autor(en) und die Quelle ordnungsgemäß nennen, einen Link zur Creative Commons Lizenz beifügen und angeben, ob Änderungen vorgenommen wurden.

Die in diesem Artikel enthaltenen Bilder und sonstiges Drittmaterial unterliegen ebenfalls der genannten
Creative Commons Lizenz, sofern sich aus der Abbildungslegende nichts anderes ergibt. Sofern das betreffende Material nicht unter der genannten Creative Commons Lizenz steht und die betreffende Handlung nicht nach gesetzlichen Vorschriften erlaubt ist, ist für die oben aufgeführten Weiterverwendungen des Materials die Einwilligung des jeweiligen Rechteinhabers einzuholen.

Weitere Details zur Lizenz entnehmen Sie bitte der Lizenzinformation auf http://creativecommons.org/ licenses/by/4.0/deed.de.

\section{Literatur}

1. Arztdata AG Arztdata AG. https://www.arztdata. de/.Zugegriffen: 30. Aug. 2020

2. Baker MW (2007) Elder mistreatment: risk, vulnerability, and early mortality. J Am Psychiatr Nurses Assoc 12:313-321

3. Bows H (2018) Sexual violence against older people: a review of the empirical literature. Trauma Violence Abuse 19:567-583

4. Büscher A (2020) Pflegewissenschaftliche Sicht. In: Suhr R, Kuhlmey A (Hrsg) Gewalt und Alter. De Gruyter, Berlin, S9-19

5. Cooper C, Selwood A, Livingston G (2009) Knowledge, detection, and reporting of abuse by health and social care professionals: a systematic review. Am J Geriatr Psychiatry 17:826-838

6. Dinkel A, Schneider A, Schmutzer G et al (2016) Die Qualität der Hausarzt-Patient-Beziehung. Patientenbezogene Prädiktoren in einer repräsentativen deutschen Bevölkerungsstichprobe. Psychother Psychosom Med Psychol 66:120-127

7. Eggert S, Sulmann D (2020) Gewalt gegen (ältere) pflegebedürftige Menschen in Deutschland - eine quantitative Annäherung. In: Suhr R, Kuhlmey A (Hrsg) Gewalt und Alter. De Gruyter, Berlin, S47-56

8. Fuertes JN, Toporovsky A, Reyes M et al (2017) The physician-patient working alliance: theory, research, and future possibilities. Patient Educ Couns 100:610-615

9. Glasman LR, Albarracin D (2006) Forming attitudes that predict future behavior: a meta-analysis of the attitude-behavior relation. Psychol Bull 132:778-822

10. Görgen T (2017) Wissen über das Phänomen Gewalt in der Pflege. In:Zentrum für Qualität in der Pflege (Hrsg) ZQP-Report: Gewaltprävention in der Pflege. Zentrum für Qualität in der Pflege, Berlin, $\mathrm{S}$ 8-12

11. Grobe TG, Dörning H, Schwartz FW (2008) GEKReport ambulant-ärztliche Versorgung: Auswertungen der GEK-Gesundheitsberichterstattung, Schwerpunkt: Erkrankungen und zukünftige Ausgaben. Asgard, St. Augustin

12. Hoffmeyer-Zlotnik JHP (2000) Regionenabgrenzungen nach wirtschaftlichen Kriterien. In: Regionalisierung sozialwissenschaftlicher Umfragedaten: Siedlungsstruktur und Wohnquartier. Springer VS, Wiesbaden, $\mathbf{S} 48$

13. Infas Institut Für Angewandte Sozialwissenschaft Gmbh (2018) Tabellenband Ärztemonitor 2018. Ergebnisse nach Facharztgruppen. In: Kassenärztlichen Bundesvereinigung (KBV) und Verband der niedergelassenen Ärzte Deutschlands e. V. (NAV-Virchow-Bund). https://www.kbv.de/ media/sp/infas_TabBand_Aerztemonitor2018 Facharztgruppen_20180615.pdf.Zugegriffen:30. Aug. 2020
14. Kassenärztliche Bundesvereinigung (2017) Bundesarztregister. In:Kassenärztliche Bundesvereinigung. https://www.kbv.de/html/ bundesarztregister.php. Zugegriffen: 30. Aug. 2020

15. KBV - Kassenärztliche Bundesvereinigung, FGW - Forschungsgruppe Wahlen Telefonfeld Gmbh (2019) Versichertenbefragung der Kassenärztlichen Bundesvereinigung 2019: 18- bis 79-jährige Befragte. März-April 2019. KBV, FGW, Mannheim

16. Kennedy RD (2005) Elder abuse and neglect: the experience, knowledge, and attitudes of primary care physicians. Fam Med 37:481-485

17. Kleina T, Horn A, SuhrRetal (2017) ZurEntwicklung der ärztlichen Versorgung in stationären Pflegeeinrichtungen - Ergebnisse einer empirischen Untersuchung. Gesundheitswesen 79:382-387

18. Kunz A, Gusy B (2005) Leitlinien in der Medizin: Anwendung, Einstellungen, Barrieren. Eine Befragung Berliner Hausärzte. Gesundheitswesen 67. https://doi.org/10.1055/s-2005-920664

19. Lachs MS, Pillemer KA (2015) Elder abuse. N Engl J Med 373:1947-1956

20. Lachs MS, Williams CS, O'Brien S et al (1998) The mortality of elder mistreatment. JAMA 280:428-432

21. Leddy MA, Farrow VA, Schulkin J (2014) Obstetrician-gynecologists' knowledge, attitudes, and practice regarding elder abuse screening. Womens Health Issues 24:e455-e464

22. Marx Y, Robra B-P, Herrmann M (2016) Priorisierungskriterien bei Polypharmazie - Ergebnisse einer schriftlichen Befragung von Hausärzten. ZAllg Med 92(6):254

23. McCreadie C, Bennett G, Gilthorpe MS et al (2000) Elder abuse: do general practitioners know or care? JRSoc Med 93:67-71

24. O'Brien JG, Riain AN, Collins C et al (2014) Elder abuse and neglect: a survey of Irish general practitioners. J Elder Abuse Negl 26:291-299

25. Pillemer K, Burnes D, Riffin C et al (2016) Elder abuse: global situation, riskfactors, and prevention strategies. Gerontologist 56(Suppl 2):S194-S205

26. Schnapp P (2018) Teilergebnisse des ZQP-Projekts Gewalt gegen Pflegebedürftige: Prävention durch Hausärztinnen und -ärzte. Psychometrische Kennzahlen verschiedener Variablen auf Basis des Pretests. Zentrum für Qualität in der Pflege, Berlin https://www.zqp.de/wp-content/uploads/Haus \%C3\%A4rztePsychometrie.pdf. Zugegriffen: 30 . Aug. 2020

27. Schnapp P, Eggert S, Suhr R (2017) Comparing continuous and dichotomous scoring of social desirability scales: effects of different scoring methods on the reliability and validity of the Winkler-Kroh-Spiess BIDR short scale. Survey methods: insights from the Field https://doi.org/ 10.13094/SMIF-2017-00002

28. Statistisches Bundesamt (2018) Pflege im Rahmen der Pflegeversicherung: Deutschlandergebnisse. https://www.destatis.de/DE/ Themen/Gesellschaft-Umwelt/Gesundheit/ Pflege/Publikationen/Downloads-Pflege/pflegedeutschlandergebnisse-5224001179004.pdf? blob=publicationFile. Zugegriffen: 30 . Aug. 2020

29. Tilden VP, Schmidt TA, Limandri BJ et al (1994) Factors that influence clinicians' assessment and management of family violence. Am J Public Health 84:628-633

30. Tourangeau R, Yan T (2007) Sensitive questions in surveys. Psychol Bull 133:859-883

31. World Health Organisation (2015) World report on aging and health. https://apps. who.int/iris/bitstream/handle/10665/186463/ 
9789240694811 eng.pdf;jsessionid=F1702B 669D1762BE957CC269E48D7198? sequence $=1$. Zugegriffen: 30. Aug. 2020

32. Yon Y, Mikton CR, Gassoumis ZD et al (2017) Elder abuse prevalence in community settings: a systematic review and meta-analysis. Lancet Glob Health 5:e147-e156
Fachnachrichten

Die Anaesthesiologie Die Innere Medizin

Die Pathologie

\section{Die Chirurgie \\ Die Kardiologie \\ eitschrift für \\ Pneumologie}

Die Dermatologie Die MKG-Chirurgie Die Psychotherapie

Die Diabetologie Die Nephrologie Die Radiologie

Die Gastroenterologie

Die

Opie

Die Unfallchirurgie

Die Gynäkologie
Die Urologie

\section{Und wenn der Facharzt eine Frau ist?}

Im Zuge einer breit angelegten Gleichstellungsinitiative des Springer Medizin Verlages werden die bisherigen, auf männlichen Berufsbezeichnungen basierenden Titel durch neue, auf die Fachgebiete bezogene Titel ersetzt. Damit die Fachzeitschriften auch außen wieder up to date sind.

Die Fachzeitschriften des Springer Medizin Verlags gehen mit den berufs- und gesellschaftspolitischen Entwicklungen und erscheinen ab ihren Juni- bzw. Juliausgaben mit neuen Titeln. Das Titelkonzept trägt auch einem geänderten Umgang mit Sprache Rechnung und verfolgt das Prinzip, dass zukünftig das Fachgebiet und nicht mehr die männliche Berufsbezeichnung im Fokus steht. So wird aus „Der Internist" ab Juni die Fachzeitschrift "Die Innere Medizin", oder „Der Chirurg“ heißt künftig „Die Chirurgie“. Diese Änderungen betreffen 21 Zeitschriften, die bei Springer Medizin erscheinen und Teil der Fachverlagsgruppe Springer Nature sind. Sie sind eine von vielen Maßnahmen innerhalb Springer Nature. Auf dem Portal Springer Medizin: www.springermedizin.de/titelupdate findet sich eine Übersicht aller betreffenden Zeitschriften samt detaillierten Erklärungen zu Motiven und Hintergründen.

Die Medizin ist schon lange nicht mehr männlich, wie es noch zu Gründungszeiten einiger Springer Fachzeitschriften der Fall war. Seit 1999 machen Frauen eine klare Mehrheit unter den Studierenden aus, im Jahr 2020 belief sich ihr Anteil auf knapp zwei Drittel. Im Berufsalltag liegt der Frauenanteil bei fast $50 \%$, in einigen Bereichen auch deutlich höher. „Diesem Wandel in der Medizin werden wir Rechnung tragen", erklärt Dr. Paul Herrmann, Director Journals und ePublishing von Springer Medizin. „Als einer der führenden Verlage in der Wissenschaftskommunikation geben wir jetzt ein klares Signal und machen Geschlech- tergerechtigkeit in der Medizin an dieser Stelle sichtbar."

Unverändert bleibt bei den Springer Fachzeitschriften die inhaltliche Ausrichtung; diese unterliegen weiterhin einer praxisrelevanten Aufbereitung und Kommunikation von Forschungsergebnissen in den Fachgebieten. Ebenso garantiert wird eine kontinuierliche Sichtbarkeit innerhalb der internationalen Wissenschaftscommunity: Ein intensiver Austausch mit relevanten internationalen Wissenschaftsdatenbanken sowie deren Offenheit und Support für das Thema garantiert, dass der Wechsel der Titel ohne nachteilige Konsequenzen verlaufen wird und eine Listung oder ein vorhandener Impact Factor bestehen bleibt.

Mehr zur Titeländerung finden Sie auf www.springermedizin.de/titelupdate oder direkt über diesen QR-Code:

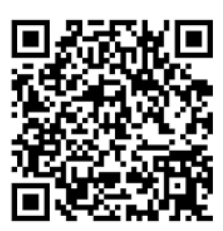

